\section{$\underset{\substack{\text { hommes } \\ \text { \& migrations }}}{ }$}

\section{Hommes \& migrations}

Revue française de référence sur les dynamiques

migratoires

$1301 \mid 2013$

Migrations et mondes ruraux

\title{
Barthélémy Toguo
}

Une écriture plastique de l'exil

\section{Isabelle Renard}

\section{(2) OpenEdition \\ 1 Journals}

\section{Édition électronique}

URL : http://journals.openedition.org/hommesmigrations/1971

DOI : 10.4000/hommesmigrations. 1971

ISSN : 2262-3353

\section{Éditeur}

Musée national de l'histoire de l'immigration

\section{Édition imprimée}

Date de publication : 1 janvier 2013

Pagination : 164-166

ISBN : 978-2-919040-21-6

ISSN : 1142-852X

\section{Référence électronique}

Isabelle Renard, «Barthélémy Toguo », Hommes \& migrations [En ligne], 1301 | 2013, mis en ligne le 29 mai 2013, consulté le 22 septembre 2020. URL : http://journals.openedition.org/hommesmigrations/ 1971 ; DOI : https://doi.org/10.4000/hommesmigrations.1971

Ce document a été généré automatiquement le 22 septembre 2020.

Tous droits réservés 


\title{
Barthélémy Toguo
}

Une écriture plastique de l'exil

\author{
Isabelle Renard
}

"En tant qu'immigrant, surtout après la chute du
mur de Berlin, j'ai réalisé combien profond est le
désir de partir, de voyager et de découvrir. L'exil
est une notion inhérente à la condition humaine
sans distinction de race ni d'origine culturelle'."
"Nous sommes tous en 'transit' permanent. Qu'un
homme soit blanc, noir, jaune, peu importe. Il est
de toute façon un être potentiellement 'exilé'."

1 La Cité nationale de l'histoire de l'immigration a acquis, en 2005 et en 2010, trois pièces majeures de Barthélémy Toguo, en résonance avec les thématiques développées par le musée.

2 Trois œuvres qui figurent, de façon métaphorique, le destin de l'exilé à travers les étapes emblématiques d'un parcours migratoire. Du départ en forme de "désir" et de "quête d'ailleurs", au passage des frontières jusqu'à l'installation dans le pays d'accueil. Road to exile ${ }^{3}$ explore la route de l'exil, sonde le chemin vers une île, peut-être "paradisiaque", signe, quoiqu'il en soit, le prélude d'une autre vie. L'artiste nous plonge dans l'épreuve du voyage. Il saisit le périple de ces migrants qui tentent la traversée en haute mer, au risque de leur vie, pour rejoindre des rives meilleures. La barque, écrasée sous la pression d'énormes baluchons multicolores renfermant des effets de fortune, vogue sur une mer fracassante de bouteilles en verre, à l'équilibre précaire. Tels les rouleaux, la houle et les lames, les bouteilles au sol rappellent les dangers du voyage alors que la pièce, dans sa démesure, pointe l'extrême fragilité de la situation et de cette embarcation qui peut, à tout instant, chavirer.

La "Barque de l'exode" se fait transposition contemporaine et géopolitique $\mathrm{du}^{4}$ "Bateau ivre" alors que résonnent encore les vers de Rimbaud : "Je sais les cieux crevant en éclairs, et les trombes / Et les ressacs et les courants [...] / Est-ce en ces nuits sans fonds que tu dors et t'exiles $[. . .]^{5}$ ?" Départ, voyages, difficultés à se déplacer, déracinement... autant de thèmes qui peuplent l'univers de l'artiste. 
Les tampons et empreintes Carte de séjour, Mamadou, France, Clandestin ${ }^{6}$, variante de l'installation The New World Climax (2001), s'inscrivent dans cette lignée et nous engagent dans une réflexion plus large sur le difficile passage de la frontière, physique, psychologique ou purement administratif.

5 Par ses tampons surdimensionnés, Barthélémy Toguo détourne avec un humour implacable les outils administratifs. Derrière la surface encrée du tampon - qui devient l'espace confié à l'artiste pour inscrire ses slogans lapidaires - se profilent des bustes humains. Têtes, cous et poitrines sont grossièrement taillés dans le bois. En regard, les empreintes correspondantes composent les pages d'un passeport fictif. Une confrontation allégorique et brutale entre l'homme et l'administration, entre celui qui prend la décision et celui qui la subit. Un coup de tampon pour résumer le statut d'une personne. Une décision administrative pour définir l'avenir du migrant, déterminer le cours d'une existence. Le tampon, cette "immense empreinte d'identité" ", devient passeur de messages tandis que les bustes, anonymes et vulnérables sous leur aspect massif, semblent en attente d'un possible ancrage.

6 Mais l'enracinement dans le pays d'accueil ne se fait pas sans difficultés comme le montre l'œuvre Climbing down ${ }^{8}$, acquise par le musée, en 2005, pour son exposition permanente. Représentation métonymique des foyers d'immigrants, la pièce traduit le hiatus profond entre rêve et réalité, promesses et désenchantement. D'emblée, le titre oxymore, Climbing down - littéralement "escalader vers le bas" ou encore, pour tenter un néologisme, "désescalader" - donne le ton. L'œuvre dans son ensemble aborde, non sans une certaine forme d'humour, les notions d'arrachement et de logement précaire. À la fin des années 1990, Barthélémy Toguo se fait l'observateur attentif de diverses manifestations sur le logement qui ont lieu à Paris et dans ses environs. Il décide de se rendre dans les quartiers où vivent les manifestants et découvre, dans certains foyers, des conditions de vie déplorables. Voulant témoigner de cette situation, l'artiste va mettre en forme le "rêve brisé" de ces hommes partis dans l'espoir d'une autre vie.

Climbing down évoque les tensions générées par la coexistence de deux espaces: un environnement partagé par tous et la sphère privée, où chacun tente de reconstruire son univers propre, balisé par des sacs et valises contenant les quelques objets emportés. Mais la frontière entre le personnel et le collectif, l'extérieur et l'intérieur est ténue.

8 L'installation, par sa taille - plus de cinq mètres de haut -, par l'entassement et la superposition gigantesque des lits (l'artiste voulait aller jusqu'à douze étages), surprend le visiteur, suscite l'étonnement, capte l'attention sur un sujet douloureux. La construction scénique de la pièce, la dimension graphique impulsée par les sacs et bagages, ne sont là que pour mieux révéler le drame et dénoncer cette "grande promiscuité austère" ", dans laquelle vivent certains immigrés. Climbing down est un choc visuel, où percent, en creux, les fêlures intimes de l'être.

9 En transit constant entre les villes de Paris, New York et Bandjoun, Barthélémy Toguo invente un univers plastique, perpétuellement nourri de ses déplacements et rencontres, où l'identité se confronte aux voyages, aux frontières, aux cultures, à l'Autre.

10 Barthélémy Toguo s'attache à évoquer les "concernés" de l'exil. Mais il est, lui-même, un artiste "concerné" qui ne cesse d'examiner l'avancement du monde, de l'interroger et de le donner à voir à travers son propre prisme. Sondant la réalité par une infinité de 
registres et de formes, il choisit toujours le médium le plus approprié pour incarner son idée et la matérialiser au plus juste. Si certaines de ses pièces sont directement inspirées par son propre parcours ou celui de populations africaines, son travail, cependant, dépasse ces expériences pour atteindre une dimension universelle. Le nomadisme de l'artiste se transmue en ligne de vie, en métaphore de la création.

11 Pour que l'art communique un sens, Barthélémy Toguo compose une œuvre poétique et politique, libre en tous les cas, où le rêve, la joie, l'humour alternent avec la souffrance. Une œuvre qui se situe dans cet espace ineffable, entre enchantement et prise de conscience.

\section{NOTES}

1. Barthélémy Toguo, in Îles jamais trouvées, dossier pédagogique, $\mathrm{n}^{\circ} 7, \mathrm{p} .15$. Dans le cadre de l'exposition Iles jamais trouvées, 18 décembre 2010-17 avril 2011, Musée d'art moderne SaintÉtienne Métropole.

2. Bartélémy Toguo, "Entretien réalisé par Arnaud Beigel", in Alexia Fabre, "Barthélémy Toguo, artiste en transit", Hommes \& Migrations, n 1261, "Accueillir autrement”, mai-juin 2006, p. 173.

3. Road to exile, 2008. Barque en bois, ballots de tissus, bouteilles. Acquisition du musée, 2010.

4. Voir aussi Julie Crenn, “J'ai deux amours, histoires d'un monde en mouvement", in Inferno, 17 novembre 2011: http://inferno-magazine.com/2011/11/17/jai-deux-amours-histoires-dunmonde-en-mouvement.

5. Arthur Rimbaud, "Le Bateau ivre" (1871), in Arthur Rimbaud, Euvre-vie, Édition du centenaire, Paris, Arléa, 1991.

6. Carte de séjour, Mamadou, France, Clandestin (Tampons et empreintes), 2010. Sculptures en bois et gravures sur bois. Acquisition du musée, 2010.

7. Voir Arnaud Beigel, in “Je reviendrai”, Parcours \#3, 2009-2010 MAC/VAL, Collection du Musée d'art contemporain du Val-de-Marne, Paris, MAC/VAL, 2009, p. 92.

8. Climbing down, 2004. Six lits superposés en bois, quatre échelles, valises, sacs multicolores. Acquisition du musée, 2005.

9. Bartélémy Toguo, "Entretien réalisé par Arnaud Beigel”, op. cit., p. 175. 